\title{
Nidos de Pholidobolus montium en un área intervenida de Mulaló, Cotopaxi-Ecuador
}

\author{
Salomón Ramírez-Jaramillo ${ }^{1,2} \square$ \\ ${ }^{1}$ Museo Ecuatoriano de Ciencias Naturales del Instituto Nacional de Biodiversidad. División de Herpetología. \\ Rumipamba 341 y Av. de los Shyris, Quito - Ecuador. \\ ${ }^{2}$ Universidad de las Fuerzas Armadas. Programa de Maestría en Sistemas de Gestión Ambiental. Av. Del \\ Progreso S/N. Sangolquí-Ecuador. \\ $\square: k p-7 s z @ h o t m a i l . c o m$
}

Recibido: 2015-11-12; aceptado: 2016-01-25

RESUMEN.- Durante 13 meses, entre septiembre del 2012 y 2013 se realizaron inspecciones a dos nidos de Pholidobolus montium, una lagartija endémica de los Andes, en una zona de intervención antrópica fuertemente agrícola. Se realizaron 6 réplicas de observación, y conteo de los huevos depositados debajo de 2 objetos en un área de $2 \mathrm{~m}^{2}$. Se registraron 69 huevos, la mayor frecuencia de puestas se observó durante los meses de septiembre, mientras que en mayo y julio no se hallaron puestas. En el seguimiento de los nidos se capturaron 17 individuos, obteniendo datos de LRC en adultos y juveniles. La densidad fue de 8 individuos $/ \mathrm{m}^{2}$ y septiembre fue el mes de mayor abundancia. Uno de los individuos capturados pertenece a P. affinis cuya simpatría se confirma en la zona. También se hallaron dos hembras grávidas, una de ellas depositó dos huevos. Adicionalmente se registraron dos individuos con malformación, uno de ellos con el carpo atrofiado.

PALABRAS CLAVES: Adaptación antrópica, densidad poblacional, lagartijas andinas, puestas de huevos.

ABSTRACT.- During thirteen months, since September 2012 until September 2013, inspections were carried out at two nests of an endemic Andean lizard, Pholidobolus montium, in a intervened area with agricultural activity. Six replicates were performed to count the eggs laid below two objects in an area of $2 \mathrm{~m}^{2}$. It was recorded 69 eggs more often during the months of September, while in May and July was not found on. In monitoring nests were captured 17 individuals, obtaining data LRC adults and youth. The density was 8 individuals $/ \mathrm{m}^{2}$ and September was the month of greatest abundance. One of the captured individuals belonging to $P$. affinis this register confirm the sympatry of this species in this area. Two pregnant females were observed, one of them laid two eggs. Additionally, two individuals with malformation were recorded, one of them presented stunted carpal.

KEYWORDS: Andean lizard, anthropic adaptation, egg laying, population density.

\section{INTRODUCCIÓN}

Pholidobolus montium (Peters 1862) es una lagartija endémica que se distribuye en los valles interandinos, desde la provincia de Cotopaxi, en el centro de Ecuador, hasta la frontera norte con Colombia, en un rango altitudinal desde los 2000 a 3190msnm (Montanucci 1973, Torres-Carvajal y Mafla-Endara 2013). Los machos son más pequeños que las hembras (Goldberg 2009), con tamaño máximo de $56 \mathrm{~mm}$ suelen presentar un color amarillo anaranjado en el pecho, mientras que las hembras alcanzan los 66mm y pueden presentar color grisáceo (Montanucci 1973). Pholidobolus montium es una especie alopátrica con restringida distribución (Montanucci 1973; TorresCarvajal y Mafla-Endara 2013), en esta localidad y más al sureste (hacia Mulaló) se encuentra en 
simpatría con P. affinis (Montanucci 1973, Hillis y Simmons 1986), no obstante son especies parapátricas (Montanucci 1973, Hillis y Simmons 1986). Son de comportamiento críptico, se refugian bajo piedras donde se mantienen seguras, a la vez que aprovechan el calor y depositan sus huevos en grupos de hasta 21. Los huevos pueden medir entre $6.6-7.6$ × $11.6-13.2 \mathrm{~mm}(7.14 \times 12.38 \mathrm{~mm} ; \mathrm{n}=9)$ (Montanucci 1973); debido a este comportamiento, sus poblaciones se encuentran comúnmente asociadas a sitios pedregosos (Montanucci 1973). De acuerdo con los criterios de la UICN (2001), y según la lista roja ecuatoriana, Pholidobolus montium está categorizada como Casi Amenazada (NT) (YánezMuñoz 2005). Debido a la escasa información que existe sobre esta lagartija andina el objetivo de este estudio es identificar épocas de anidación mediante evaluaciones temporales dentro de una área agropecuaria cercana a Mulaló.

\section{METODOLOGÍA}

Área de estudio: La parroquia de Mulaló $\left(78^{\circ} 35^{\prime}\right.$ $34.9^{\prime \prime} \mathrm{W} 0^{\circ} 45^{\prime} 58.4^{\prime \prime} \mathrm{S}$ ) a $3004 \mathrm{msnm}$ (Figura 1), se ubica al sur del Nudo de Tiopullo, cerca del Volcán Cotopaxi, pertenece a la provincia de Cotopaxi, cantón Latacunga. La zona de estudio pertenece al ecosistema de Arbustal Siempreverde Montano del norte de los Andes (MAE 2013). En general su cobertura vegetal natural está destruida casi en su totalidad y fue remplazada por cultivos de brócoli, rosas y pasto para ganado, incluyendo plantaciones de Eucalyptus globulus (eucalipto) y Pinus radiata (pino), los remanentes de bosque nativo se encuentran en sitios poco accesibles y presentan una vegetación xerofítica, con pocas hierbas y pequeños arbustos (Valencia et al. 1999).

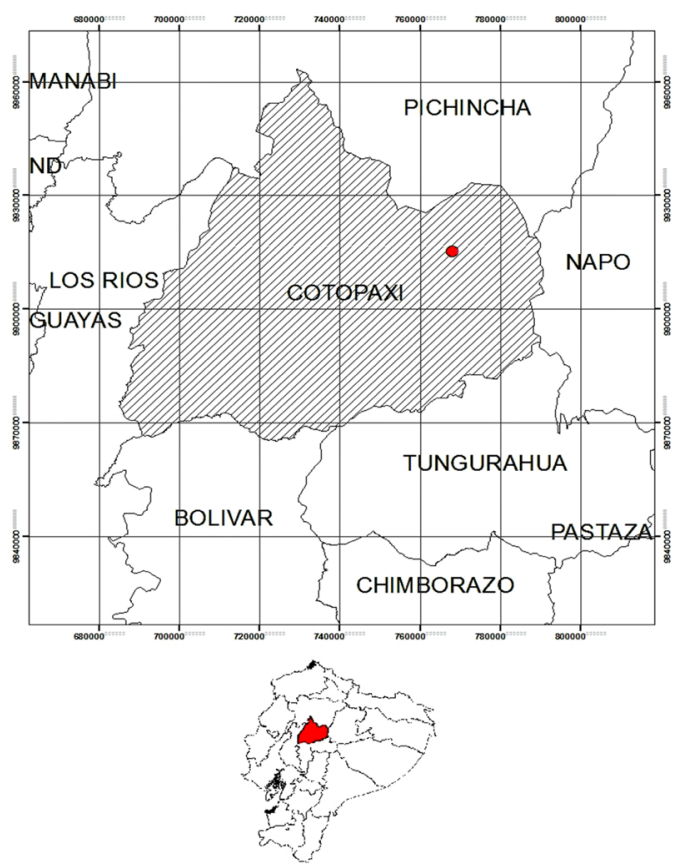

Figura 1. Mapa de ubicación de los nidos de P. montium.
Muestreos: En agosto de 2012 se realizó el reconocimiento del área y selección del sitio de muestreo. Los muestreos de campo se realizaron durante los meses de septiembre de 2012, enero, abril, mayo, julio y septiembre de 2013, dedicando dos días de muestreo en cada mes. La técnica de muestreo por parcheshademostradoser efectiva para determinar el número de individuos, abundancia relativa y densidad de lagartijas (Sánchez 2011). En este caso, el área de muestreo consistió en un parche de $2 \mathrm{~m}^{2}$ donde se hallaba una piedra y un bloque de construcción ( $23 \times 12 \times 10 \mathrm{~cm})$. Las inspecciones tenían una duración de 7 a 9 minutos diarios, con un total aproximado de 90 minutos/hombre de esfuerzo de muestreo.

Los huevos registrados fueron contabilizados en su sitio (no fueron marcados), únicamente en el primer muestreo se midieron 16 huevos. No se colectaron especímenes testigo, pero se tomaron registros fotográficos y las siguientes medidas morfométricas: Longitud Rostro-Cloaca (LRC) y Longitud de la Cola (LC).

Análisis de datos: Las medidas de LRC y LC, se analizaron con promedios de medidas máximas y mínimas. Según Goldberg (2009), se categorizó la LRC de los adultos a partir de 10 s.7cm, valores menores corresponden a juveniles. Para estimar la densidad poblacional se aplicó una prueba de estimación de parámetros utilizando el programa BioEstat 5.0. Se tomó en cuenta el área próxima de observación $\left(10000 \mathrm{~m}^{2}\right)$, el área de muestreo $\left(2 \mathrm{~m}^{2}\right)$, el número de muestras (1) y el número de individuos registrados (16). La época lluviosa en esta zona va de octubre a junio y la época seca desde julio hasta septiembre (INAMHI, 2014).

\section{RESULTADOS Y DISCUSIÓN}

Puestas de huevos y nidos: Se registraron 69 huevos, con un mayor número de registros en septiembre del 2012, mientras que en mayo y julio del 2013 no hubo registros (Tabla 1). El tamaño de los huevos $(\mathrm{n}=16)$ fue de $6.6-9 \mathrm{~mm}(7.37 \pm 1.09 \mathrm{~mm})$ de ancho y de $10.9-14.1 \mathrm{~mm}(12.52 \pm 1.08 \mathrm{~mm})$ de largo. Existe una correlación negativa entre el largo y el ancho de los huevos $(\mathrm{r}=-0.35)$. Durante la época seca se registraron 43 huevos, mientras que en la época lluviosa fue de 26 huevos (Figura 2).

Las puestas de huevos fueron mayores durante la época seca $(62.3 \%)$; sin embargo, los registros en época lluviosa se mantienen, lo que sugiere una reproducción continua a lo largo del año, y con mayor frecuencia durante el mes de octubre (Bustard 1964, Montanucci 1973, Goldberg 2009). 
Tabla 2. Meses de muestreo y sus registros por nido de Pholidobolus montium.

\begin{tabular}{lcccc}
\hline Fecha & \multicolumn{2}{c}{$\mathbf{N}^{\circ}$ huevos } & \multicolumn{2}{c}{$\mathbf{N}^{\circ}$ individuos } \\
\hline Nep-12 & Nido 1 & Nido 2 & Nido 1 & Nido 2 \\
Jan-13 & 8 & 9 & 0 & 1 \\
Apr-13 & 8 & 5 & 2 & 1 \\
May-13 & 0 & 5 & 2 & 0 \\
Jul-13 & 0 & 0 & 1 & 1 \\
Sep-13 & 5 & 4 & 2 & 2 \\
TOTAL & $\mathbf{4 6}$ & $\mathbf{2 3}$ & $\mathbf{9}$ & $\mathbf{8}$
\end{tabular}

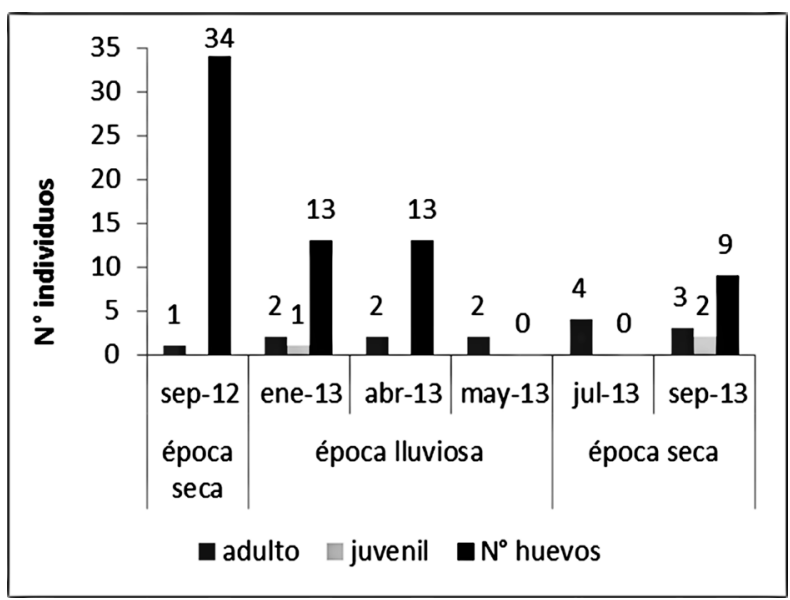

Figura 2. Dinámica poblacional y de puestas de huevos de Pholidobolus montium.

Las puestas requieren de condiciones adecuadas para su sobrevivencia, el lugar de oviposición, el aumento o disminución de temperatura y la humedad son factores determinantes para el éxito de las puestas (Bustard 1964). Por lo tanto, el microhábitat elegido para la puesta (Figura 3A y 3B), debe brindar estas características durante ambas épocas con el fin de evitar que los huevos se sequen (Figura 3C).

Los nidos fueron hallados en grupos de 2 a 13 huevos (Figura 3C, 3D y 3E). Las hembras colocan los huevos individualmente en grupos específicos y al parecer existe cierto tipo de cuidado parental pues se observó a una hembra reposando a $27 \mathrm{~cm}$ de una puesta (Figura 3F). En agosto 2013 una hembra capturada (Figura 4A) depositó dos huevos en un lapso de dos días, este número concuerda con lo mencionado por Bustard (1964) y Montanucci (1973). El tamaño de los huevos es similar a los datos registrados por Montanucci (1973), aunque en este caso los huevos son más pequeños y anchos, en contraste las mediciones de Bustard (1964) indican huevos más pequeños y delgados $(10-12 \mathrm{~mm} \mathrm{x}$ 6-7mm). Bustard (1964), observó la incubación de un huevo durante 216 días luego de lo cual reportó el crecimiento del huevo hasta casi el doble de su tamaño original. Al inicio los huevos son de un color blanquesino y posteriormente se obscurecen, su textura es semirrígida y con apariencia de plástico cuando se secan o después de la eclosión.

Se observó que esta lagartija es muy sensible ante la presencia humana, durante el primer muestreo se encontraron 34 huevos mientras que en los siguientes muestreos la variación fue de 0-13 huevos. Se desconoce si la misma hembra realiza varias puestas en el mismo nido, aunque se pudo notar que varias hembras depositaron sus huevos en los mismos sitios (Figura 3). Los huevos son enterrados a menos de $2.5 \mathrm{~cm}$, de tal modo que se agrupan creando un microhábitat entre las raíces, la tierra y las piedras, brindándoles refugio y protección. Cuando llueve mucho o el clima está muy frío, las puestas pueden resguardarse temporalmente mientras la piedra o bloque mantienen el calor acumulado durante el día; de igual manera, cuando el clima es muy seco los huevos reciben la humedad suficiente para que puedan desarrollarse (Montanucci 1973).
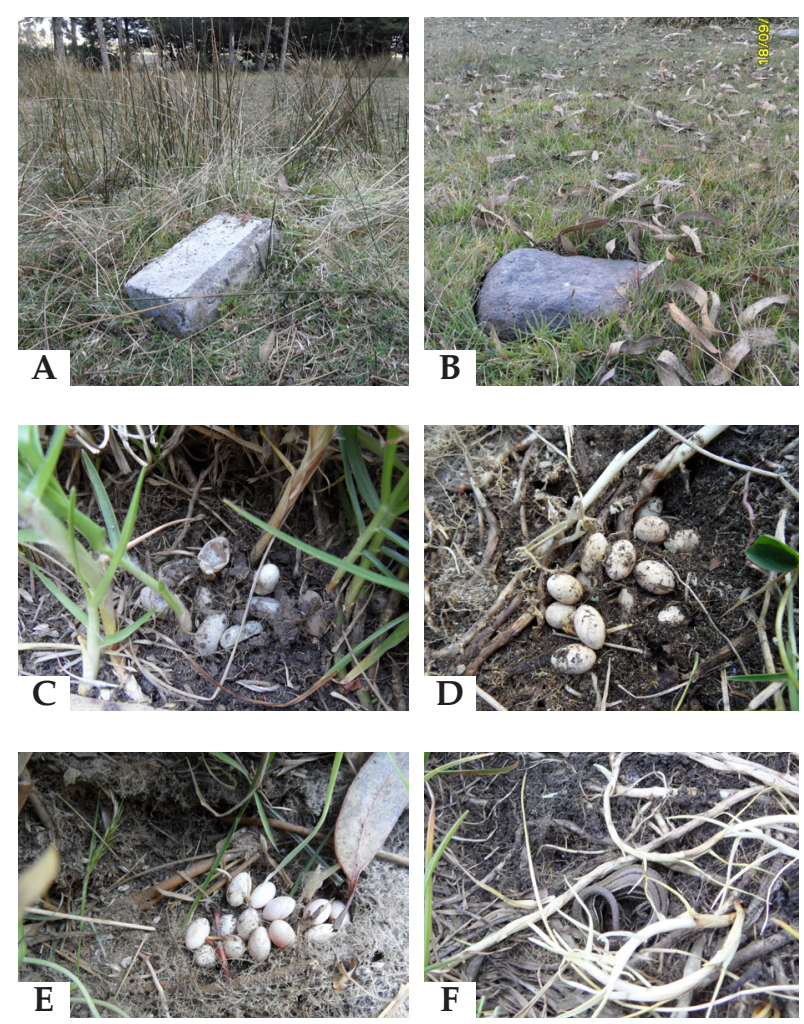

Figura 3. Durante la primera inspección realizada el 18-09-2012, se registra en el sitio de estudio, A) bloque de construcción, parte del nido. B) piedra usada como parte del nido. C) puesta de 9 huevos (8 secos y 1 normal). D) puesta con 12 huevos. E) puesta con 13 huevos. F) posición de hembra grávida descansando o cuidando las puestas a $27 \mathrm{~cm}$ de distancia de una puesta de 9 huevos. 
Población y densidad.- Se registraron 17 individuos agrupados en 14 adultos y tres juveniles. En el mes de julio de 2013 se realizó la mayor cantidad de registros (4 individuos) y en septiembre de 2012 el menor número (1 individuo), los juveniles fueron poco avistados (Figura 2). Durante la época seca se observaron 10 individuos (ocho adultos y dos juveniles), mientras que en la época lluviosa fueron encontrados siete individuos (seis adultos y un juvenil) (Figura 2). Se observaron dos hembras grávidas, una en septiembre de 2012 (Figura 3F) y otra en julio de 2013 (Figura 4A) (LRC= 4.97-6.99 y $L C=10.38-12.38 \mathrm{~cm})$. La densidad poblacional estimada es de 8 individuos/m2 (80000 ind/Ha).

El tamaño estimado para Pholidobolus montium a partir de los 17 individuos capturados fue de $4.7 \pm 1.31 \mathrm{~cm}$ para la LRC y entre $8.13 \pm 1.44 \mathrm{~cm}$ para LC. El $58.8 \%$ de los individuos fue registrado durante la época seca, lo que sugiere que en esta temporada existe mayor actividad y frecuencia al microhábitat. En este estudio no se pudo identificar con exactitud a las hembras (debido a que no fueron sacrificadas), sin embargo fue fácil identificarlas cuando estaban grávidas (Figura 3F y 4A). La relación entre LRC y LC, es de 1:1.6; la cola es usada como protección y camuflaje durante su descanso así como para el cuidado parental (Figura 3F), en casos de predación la cola es separada para engañar al depredador y permitir el escape. Para medir el grado de predación es necesario registrar la mutilación de colas o extremidades, durante este estudio se registraron a dos individuos con cola regenerada (Figura 4B y 4C).

El valor estimado de la densidad poblacional (80 000 ind/Ha) se considera excesivo, y puede estar influenciado por el muestreo, hábitat, microhábitat y tipo de intervención antrópica. Es fácil encontrar individuos en microhábitats que les brindan refugio y protección, así como en las horas de mayor actividad lo cual puede incrementar el número de registros. Para determinar el tamaño poblacional son necesarios estudios temporales en los cuales se emplee marcaje para captura-recaptura de individuos (Sánchez 2011).

Uno de los individuos registrados en mayo de 2013 perteneció a Pholidobolus affinis ( $\mathrm{LRC}=5.4 \mathrm{y} \mathrm{LC}=$ $4.1 \mathrm{~cm}$ ), este registro concuerda con lo reportado por Montanucci 1973 y Hillis y Simmons 1986 y confirma la simpatría entre estas dos especies.

Adaptación antrópica.- El área de estudio se encuentra dentro de una zona agrícola y ganadera, próxima a una acequia y rodeada con poca vegetación nativa; cuenta con aproximadamente cuatro hectáreas de vegetación herbácea usadas para pastoreo ocasional, rodeado de eucalipto
(Eucaliptus globosus), pino (Pinus radiata) y ciprés (Cupressus sempervirens). El parche de muestreo se hallaba entre 13 a $30 \mathrm{~m}$ de distancia de pinos y cipreses (Figura 3A y 3B), lo que evidencia que los individuos presentan cierta tolerancia a estas especies introducidas y tipo de hábitat. En dos nidos inspeccionados se observó la disminución de la cantidad de puestas, lo que hizo evidente la sensibilidad ante la actividad humana; aunque la disponibilidad del recurso (en este caso el bloque y la piedra) podría ser un factor que incremente la probabilidad de encontrar más individuos (Montanucci 1973). Durante las inspecciones se registraron dos individuos con malformaciones (Figura 4B y 4C), uno con un abultamiento al final de la cola producida por la regeneración de la misma (Figura 4B) y otro con el carpo y sus falanges atrofiadas con (Figura 4C).
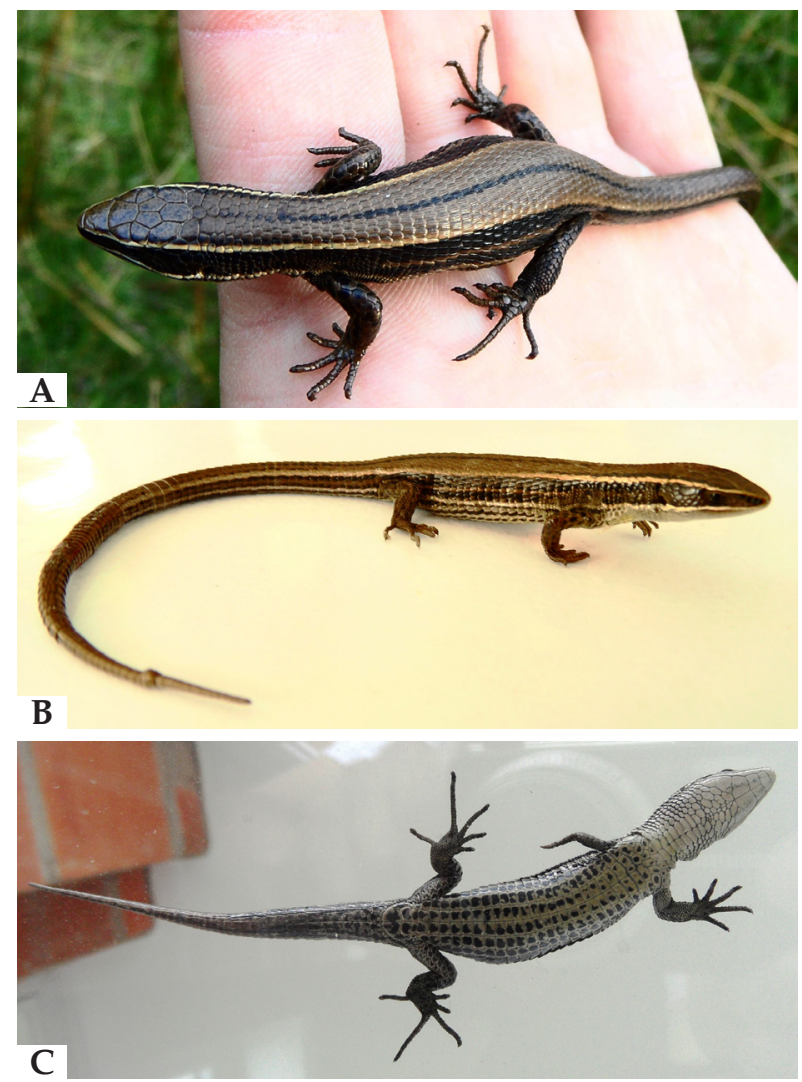

Figura 4. A) hembra grávida hallada el 08-07-2013. B) individuo con cola abultada 17-09-2013. C) individuo con malformación en la extremidad superior derecha 17-04-2013.

\section{AGRADECIMIENTOS}

A la Fundación Sierra Flor por abrirme las puertas y darme la oportunidad de ser parte de su proyecto de educación. Gracias a Jacky Barreiro y Viviana Tantardini por darme la apertura y motivación para poder realizar mis prácticas de investigación en campo; de igual forma a Alicia González y Joan Rovira por su motivación y colaboración durante 
mi estancia. A Mario Yánez, Juan Pablo y Carolina Reyes por sus sugerencias y comentarios. A Mauricio Ortega por su revisión al primer borrador y a Mauricio Herrera por su ayuda con el abstract.

\section{BIBLIOGRAFÍA}

Bustard R. 1964. Egg laying and incubation of the striped mountain lizard Pholidobolus montium (Teiidae) with notes oa an incubator. British Journal of Herpetology 3: 163-164.

Goldberg SR. 2009. Note on Reproduction of Pholidobolus montium (Squamata: Gymnophthalmidae) from Ecuador. Bulletin of the Chicago Herpetological Society 44(11):167-168.

Hillis DM. 1985. Evolutionary genetics of the andean lizards genus Pholidobolus (Sauria: Gymnophthalmidae): phylogeny, biogeography, and a comparison of tree construction techniques. Systematic Zoology 34(2): 109-126.

Hillis DM y Simmons J E. 1986. Dynamic change of a zone of parapatry between two species of Pholidobolus (Sauria: Gymnophthalmidae). Journal of Herpetology 20 (1): 85-87.

Instituto Nacional de Meteorología e Hidrología (INAMHI), 2014. Anuario Metereológico Nro. 51-2011. Quito-Ecuador. 149 pp.

Ministerio del Ambiente del Ecuador (MAE). 2013. Sistema de Clasificación de los Ecosistemas del Ecuador Continental. Subsecretaría de Patrimonio Natural. Quito. 235 pp.

Montanucci RR. 1973. Systematics and evolution of the Andean lizard genus Pholidobolus (Sauria: Teiidae). University of Kansas, Museum of Natural History Miscellaneous Publication 59: $1-52$.

Sánchez O. 2011. Evaluación y monitoreo de poblaciones silvestres de reptiles. En: Sánchez O, Zamorano P, Peters E y H Moya (eds). Temas sobre conservación de vertebrados silvestres en México: 83-120. Secretaría de Medio Ambiente y Recursos Naturales-Instituto Nacional de Ecología (INE-Semarnat). México, D.F., México.

Torres-Carvajal O y Mafla-Endara P. 2013. Evolutionary history of Andean Pholidobolus and Macropholidus (Squamata: Gymnophthalmidae) lizards. Molecular Phylogenetics and Evolution 68: 212-217.
Valencia R, Cerón C, Palacios W y Sierra R. 1999. Las formaciones naturales de la Sierra del Ecuador. En: Sierra R (eds.). Propuesta Preliminar de un Sistema de Clasificación de Vegetación para el Ecuador Continental: 79-108. Proyecto Instituto Ecuatoriano Forestal y de Áreas Naturales y Vida Silvestre/Global Environment Facility-Banco Internacional de Reconstrucción y Fomento, Quito-Ecuador.

Yánez-Muñoz, 2005. Lagartija minadora (Pholidobolus montium). En: Carrillo E, Aldás S, AltamiranoBenavides MA, Ayala-Varela F, Cisneros-Heredia, DF., Endara A, et al. 2005. Lista roja de los reptiles del Ecuador: 27. Fundación Novum Milenium, UICN-Sur, UICN-Comité Ecuatoriano, Ministerio de Educación y Cultura, Serie Proyecto Peepe, Quito, Ecuador, 46 pp. 